\title{
Scaling Properties in Highly Anisotropic Systems
}

\author{
Qiming $\operatorname{Li}^{1}$, S. Katsoprinakis, ${ }^{2}$, E. N. Economou ${ }^{2}$, C. M. Soukoulis ${ }^{1,2}$ \\ ${ }^{1}$ Ames Laboratory and Department of Physics and Astronomy, \\ Iowa State University, Ames, IA 50011 \\ ${ }^{2}$ Research Center of Crete and Department of Physics, University of \\ Crete, P. O. Box 1527, 71110 Heraklion, Crete, Greece
}

\begin{abstract}
Scaling of the conductances and the finite-size localization lengths is generalized to anisotropic systems and tested in two dimensional systems. Scaling functions of isotropic systems are recovered once the dimension of the system in each direction is chosen to be proportional to the localization length. It is also shown that the geometric mean of the localization lengths is a function of the geometric mean of the conductivities. The ratio of the localization lengths is proportional to the square root of the ratio of the conductivities, which in turn is proportional to the anisotropy strength $t$, in the weak scattering limit.
\end{abstract}

PACS numbers: 72.15.Rn $\quad$ 71.30.+h $\quad$ 74.25.Fy

Scaling theories have been successfully applied to the problem of Anderson localization [1.2] involving the effects of disorder on the nature of the electronic wave function. The most remarkable result of the scaling theory is its prediction [3] that a continuous metal-insulator transition exists in three dimensions, and all the states are localized in two dimensions, in the absence of magnetic field and spin-orbit interaction. The essential hypothesis of the one parameter scaling theory of localization is that the rate of change of the conductance when the size of the system changes is controlled by the conductance alone. The critical conductance $G_{c}$ that separates true metals from insulators is estimated [1] to be $G_{c}=0.1 \frac{e^{2}}{\hbar}$. The scaling function should also be universal within a class that is characterized by a few general symmetries of the governing Hamiltonian. The scaling theory results are supported by a large number of numerical studies [2] in $d=2$ and $d=3$. Most notably, finite size scaling calculations [4] on the transmission properties of a quasi-one dimensional system explicitly demonstrated the existence of a universal scaling function close to the critical regime.

Most of the previous work involves isotropic systems. Recently, the problem of Anderson localization in anisotropic systems has attracted considerable attention [5, 8, largely due to the fact that a large variety of materials are highly anisotropic. It was recently shown [6] that in a highly anisotropic system of weakly coupled planes, states are localized in the direction parallel and perpendicular to the plane at exactly the same amount of critical disorder, in support of the one parameter scaling theory which excludes the possibility of having a wave function localized in one direction and extended in the other two. However, several issues regarding the relation between the conductances in different directions were raised. Most importantly, the question of scaling of conductances and localization lengths was not resolved [6]. Although anisotropy is known not to change the universality and thus the critical behavior of the system [9], the exact form of the scaling function, on the other hand is expected to depend on the anisotropy in the form of anisotropic physical parameters such as anisotropic hopping integrals or geometrical aspect ratios [10].

Extending the scaling argument to an anisotropic system, we assume that the logarithmic derivative, $\beta_{i}$, of the dimensionless conductance $g_{i}$, in any direction will be a function of the conductance in that direction as well as other directions,

$$
\beta_{i}=\frac{d \log g_{i}}{d \log a}=\beta_{i}\left(\left\{g_{i}\right\}\right)
$$

where $a$ is an appropriate length scale. All the $g_{i}$ become relevant scaling parameters. All other physical quantities, such as anisotropic hopping integrals or anisotropic geometrical shapes, should enter only through the conductances $g_{i}$. Exactly the same argument can be applied to the scaling function of localization length, obtained from transfer matrix calculations with a quasi-one dimensional geometry of cross section $M_{j} \times M_{k}$,

$$
\frac{\lambda_{i}\left(M_{j}, M_{k}\right)}{\xi_{i}}=h\left(\frac{M_{j}}{\xi_{j}}, \frac{M_{k}}{\xi_{k}}\right)
$$

where $\lambda_{i}$ is the finite size localization length in the direction $i$ and $\xi_{l}(l=1,2,3)$ is the localization length for the infinite system. The fundamental assumption in Eq. (2) is that localization lengths provide the only characteristic length scale. Once the characteristic lengths are measured in terms of the localization lengths in the corresponding directions, the scaling behavior of the system within the same universality class, are governed by the same equation.

The scaling functions $\beta_{i}$ and $h$ describe the behavior of both systems with isotropic Hamiltonians but non-cubic geometry as well as systems with anisotropic Hamiltonians. Scaling in anisotropic systems in general is not known. Only when the conductances in all directions are 
the same, then the scaling function $\beta_{i}$ will be exactly the same as that of a cubic isotropic system. For an anisotropic system, this can only be achieved by choosing an appropriate geometry which might not be known a priori. As an example we will see that indeed such a procedure works in a system with highly anisotropic hopping. We will demonstrate that in two dimensional systems, Eq.(2) can be applied straightforwardly such that all the data are described by the scaling functions of the isotropic system. Furthermore, we will also show that the geometric mean of the localization lengths is a universal function of the geometric mean of the bare conductivity, and their ratio can also be estimated in the weak scattering limit. These results follow directly from applying the basic idea of scaling theory.

We consider the following Hamiltonian for an anisotropic $2 \mathrm{~d}$ disordered model

$$
H=\sum_{n} \epsilon_{n}|n><n|+\sum_{n, m} t_{n m}|n><m|
$$

where $\mathrm{n}$ labels the sites of a square lattice. The on-site energies $\epsilon_{n}$ are independently distributed at random, within an interval of width $\mathrm{W}$. The second term is taken over all pairs of nearest neighbor (n.n.) sites, and the hopping integral $t_{n m}=1$ or $\mathrm{t}(<1)$, depending on hopping directions. As a convention, we have assigned the direction with the large $\left(t_{n m}=1\right)$ and the small $\left(t_{n m}=t\right)$ hopping value as the parallel $(\|)$ and the perpendicular $(\perp)$ directions, respectively.

In two dimensional systems, Eq. (2) can be written as

$$
\frac{\lambda_{i}\left(M_{j}\right)}{M_{j}}=\frac{\xi_{i}}{\xi_{j}} f\left(\frac{M_{j}}{\xi_{j}}\right)
$$

where $f(x)=h(x) / x$ is the scaling function for isotropic systems. We have used the transfer matrix method [2] to calculate the finite size localization length $\lambda_{i}\left(M_{j}\right)$ for many $M_{j}(i, j=1,2)(\mathrm{M}=24,48,96,120,150,300)$ and $\mathrm{W}=2-14$ and several $\mathrm{t}$ and $\mathrm{E}$, for both directions. Figure 1 shows that all of our raw numerical data for both $\lambda_{M}^{\|}$ and $\lambda_{M}^{\perp}$ for different anisotropies t different disorder $\mathrm{W}$ and different energies $\mathrm{E}$ follow one universal curve, by appropriately choosing the localization length in the two directions, $\xi_{\|}$and $\xi_{\perp}$. The solid line through the data in Fig. 1, is the 2 d isotropic scaling function. This is a direct confirmation of the scaling relation Eq. (4).

An important consequence of Eq. (4) is that at the critical point, if any, the geometric mean of the ratio of the finite-size localization length to the cross-section width is a constant. This was indeed found [6] to be true but interpreted instead as a result of possible conformal invariance. We point out that at the critical point,the geometric mean of the conductances along the different directions may not be a constant. This behavior of the conductances is different from that of $\lambda_{M} / M$ and needs further study for its complete understanding.

To further test the scaling idea we have calculated the conductance $G$ in the two different directions for our anisotropic system. From the Multichannel Landauer formula [1],12], $G=\frac{e^{2}}{h} \operatorname{Tr}\left(t^{\dagger} t\right)$, where $\mathrm{t}$ is the transmission matrix. With anisotropic hoppings, one should choose a geometry other than the square such that the conductance is the same in all the directions and then scale up the size of the system [8]. The conductance should remain isotropic if one parameter scaling theory is correct [5]. We have tested this idea in a $2 \mathrm{~d}$ system with $t=0.1$. The ratio of the two localization lengths was found to be 10 at $\mathrm{W}=3.6$. We have scaled up the system of a rectangle of size $\mathrm{M} \times \mathrm{N}$ by a factor 4 , and from Fig. 2, one can clearly see that although the conductance becomes extremely small it remains isotropic, in agreement with the predictions of the one parameter scaling theory [5]. For a square geometry and with the same parameters as in Fig. 2, the conductances in the two directions would diverge rapidly as the system size scales up.

Another length rescaling aspect can be seen by considering the self-consistent theory of localization. It was shown in an earlier work [6] that in order the localization criteria to be the same in all directions, the length scale has to be chosen proportional to the square root of the bare conductivity. This leads to an equation for the metal-insulator transition that is exactly the same as that of the isotropic system except that both the bare conductivity and the effective lattice constant are replaced by their geometric means. A direct consequence of this formulation is that the geometric mean of the localization (or correlation) lengths should be only a function of the geometric mean of the bare conductivities, ie,

$$
<\xi>_{g}=f_{l}\left(<\sigma_{0}>_{g}\right) / S_{f},
$$

where $<>_{g}$ denote the geometric mean of the values in the two directions. $\sigma_{0}$ is the bare conductivity and $S_{f}$ is the Fermi surface area that enters through the relation $\sigma_{0} \sim S_{f} \ell$. $\ell$ is the mean free path. $f_{l}$ is a function that can be obtained via the Potential Well Analogy(PWA) or the self consistent theory of localization. Using the PWA Ref.13 obtained $\xi=2.72 \ell \exp \left[\pi^{2} \hbar \sigma_{0} / e^{2}\right]$.

Eq. (5) can be easily checked in the weak disorder limit, at which the geometric mean of the bare conductivity can be shown to be $\bar{\sigma}_{0}=15 \sqrt{2} t / \pi W^{2}$, within the Coherent Potential Approximation (CPA)[14]. For the $2 \mathrm{~d}$ anisotropic system, $S_{f}(E=0)=4 \pi \sqrt{1+t^{2}}$. We have plotted $S_{f}<\xi_{\perp} \xi_{\|}>^{1 / 2}$ versus $15 \sqrt{2} t / \pi W^{2}$, and find that the data fall into one universal curve for all the different anisotropies $\mathrm{t}$ and disorder $\mathrm{W}$. This weak scattering limit behavior of the geometric mean of the localization lengths versus the geometric mean of the conductivities is very suggestive of the way the localization lengths have to scale. The full expression, valid for all disorder strength,

$$
\sigma_{i 0}=\frac{2 e^{2} \hbar}{\pi} \sum_{k} v_{i}^{2}(k) \frac{\Sigma_{2}^{2}}{\left[\left(E-\Sigma_{1}-E(k)\right)^{2}+\Sigma_{2}^{2}\right]^{2}}
$$


can also be evaluated. $\Sigma=\Sigma_{1}-i \Sigma_{2}$ is the self-energy obtained by solving a self-consistent equation [5, 13, 15. This shows remarkable good scaling as shown in Fig. 3, including results for $\mathrm{E}=0$, as well as for $E \neq 0$. The curve in Fig. 3 shows how the geometric mean of the localization length depends on the geometric mean of the bare conductivity in a universal fashion, independent of the anisotropy, energy and disorder. These results are a strong confirmation of scaling in anisotropic systems. Notice that the geometric mean of the conductivities $<$ $\sigma_{0}>_{g}$, and not of the conductances, is the appropriate quantity that gives the same results as in the isotropic case. It is therefore appropriate that $\left\langle\sigma_{0}\right\rangle_{g}$ will be used in the interpretation of experiments in highly anisotropic systems.

The ratio of the localization lengths can be obtained by carrying the length rescaling idea further. We can see that the conductances in all the directions should be the same, if the dimension of the system is proportional to the localization length in that direction. This implies the following relation

$$
\frac{\xi_{i}}{\xi_{j}}=\left(\frac{\sigma_{i}}{\sigma_{j}}\right)^{1 / 2}=\left(\frac{\sigma_{i 0}}{\sigma_{j 0}}\right)^{1 / 2}\left(\frac{\alpha_{i}}{\alpha_{j}}\right)^{1 / 2}
$$

$\sigma_{i}$ is the exact value of the conductivity, $\sigma_{i 0}$ is the bare conductivity which can be calculated within the CPA, $\alpha_{i}$ is the correction of the bare conductivity in the i direction. It is very difficult to calculate the correction $\alpha_{i}$, but it approaches one in the weak scattering limit. In Fig. 4 , we show the results of $\xi_{\perp} / t \xi_{\|}$versus $1 / \mathrm{W}$ for different anisotropies $t$ and energies E. In the weak disorder limit, we can approximate $\sigma$ by $\sigma_{0}$, and this is shown as open symbols in Fig. 4. Notice that in the weak disorder limit, $\mathrm{W} \rightarrow 0$, and for $\mathrm{t} \rightarrow 0, \sigma_{0 \perp} / \sigma_{0 \|} \sim t^{2}$ and by using Eq. (7), one obtains for the ratio of the localization lengths ,$\xi_{\perp} / \xi_{\|} \sim t$. This behaviour is clearly seen in Fig. 4 for large $1 / \mathrm{W}$. Agreement with the CPA results for the conductivity are excellent for weak disorder. Deviation of the ratio from the open symbols for strong disorder indicates that the true conductivity at length scale $\xi$ is stongly normalized compared with the bare conductivity. However, it is notable that the trend of the ratio as $\mathrm{W}$ increases is captured by the simple expression. For large $\mathrm{W}$, no dependence on E should be expected for small E, thus the ratios converge to the same value for different $\mathrm{E}$ with $\mathrm{t}=0.3$, as can be seen in the insert in Fig. 4 .

In summary, we have performed an extensive numerical study of the scaling properties of highly anisotropic systems. Scaling functions of isotropic systems are recovered once the dimension of the system in each direction is chosen to be proportional to the localization length.In the localized regime, the ratio of the localization lengths is proportional to the square root of the ratio of the conductivities which in turn is proportional to the strength of the anisotropy t (i.e. $\xi_{\perp} / \xi_{\|} \sim t$ ). Recall that in the extended regime [5,6] the ratio of the correlation lengths is proportional to the ratio of the conductivities (i.e. $\xi_{\perp} / \xi_{\|}$ $\left.=\sigma_{0 \perp} / \sigma_{0 \|} \sim 1 / t^{2}\right)$. It was also shown that the geometric mean of the localization lengths is a function of the geometric mean of the conductivities, not of the conductances. Finally, it was numerically shown that the conductances along the two different directions of the anisotropic system are the same, provided that the dimension of the anisotropic system is proportional to the localization length in this direction. This procedure can be easily used in other anisotropic systems.

Ames Laboratory is operated for the U.S. Department of Energy by Iowa State University under Contract No. W-7405-Eng-82. This work was supported by the Scalable Computing Laboratory of Ames Lab, the director for Energy Research, Office of Basic Energy Sciences, NATO Grant No. CRG 940647. This work was also supported in part by EU grants and a חENE $\Delta$ Research Grant of the Greek Secretariat of Science and Technology.

[1] For review, see P. A. Lee and T. V. Ramakrishnan, Rev. Mod. Phys. 57, 287 (1985).

[2] For a recent review, see B. Kramer and A. Mackinnon, Rep. Progr. Phys. 56, 1469 (1993).

[3] E. Abrahams, P. W. Anderson, D. C. Licciardello and T. V. Ramakrisham, Phys. Rev. Lett. 42, 673 (1979).

[4] A. MacKinnon and B. Kramer, Phys. Rev. Lett. 47, 1546 (1981).

[5] P. Wölfle and R. N. Bhatt, Phys. Rev. B 30, 3542 (1984); R. N. Bhatt, P. Wöffle, and T. V. Ramakrishnan, ibid. 32, 569 (1985); D. Vollhardt, and P. Wölfle, in Electronic Phase Transitions, ed. by W. Hanke and Yu V. Kopaev (Elsevier, New York, 1992), Ch. 1, p. 38.

[6] Qiming Li, C. M. Soukoulis, E. N. Economou, and G. S. Grest, Phys. Rev. B 40, 2825 (1989); I. Zambetaki, Qiming Li, E. N. Economou, and C. M. Soukoulis, Phys. Rev. Lett. 76, 3614 (1996).

[7] W. Xue, P. Sheng, Q. J. Chu, and Z. Q. Zhang, Phys. REv. Lett. 63, 2837 (1989); Phys. Rev. B 42, 4613 (1990); Q. J. Chu and Z. Q. Zhang, ibid. 48, 10761 (1993).

[8] W. Apel and T. M. Rice, J. Phys. C 16, L1151 (1983).

[9] V. Privman and M. E. Fisher, Phys. Rev. B 30, 322 (1984).

[10] C.-K. Hu, C.-Y. Lin and J.-A. Chen, Phys. Rev Lett. 75, 193 (1995), and Physica A 221, 80 (1995).

[11] J. L. Pichard, N. Zanon, Y. Imry, and A. D. Stone, J. Phys. (France)51, 587 (1990).

[12] E. N. Economou and C. M. Soukoulis, Phys. Rev. Lett. 46, 618 (1981); D. S. Fisher and P. A. Lee, Phys. Rev. B 23685 (1981).

[13] E. N. Economou and C. M. Soukoulis, Phys. Rev. B 28, 1093 (1983); ibid. 30, 1686 (1984); A. D. Zdetsis, C. M. Soukoulis, E. N. Economou, and G. S. Grest ibid 32, 7811 (1985).

[14] In the weak scattering limit, the static conductivity $\sigma_{i 0}$ is given [6,13] by $\sigma_{i 0}=e^{2} S<v_{i}^{2} / v>\tau / 4 \pi^{2}$, where i 
$=\mathrm{x}$ and $\mathrm{y}$ and $\mathrm{S}(\mathrm{E})$ is the area of the surface $E(\vec{k})=$ $E=2 \cos k_{x} a+2 t \cos k_{y} \alpha$ and the average is taken over this surface. Normally $\mathrm{S}(\mathrm{E})$ is tedious to calculate, however for $\mathrm{E}=0$, one obtains $S(E=0)=4 \pi \sqrt{1+t^{2}}$. The $\left.\mathrm{S}(\mathrm{E})<v_{i}^{2} / v\right\rangle$ can be expressed [6,13] in terms of the lattice Green's function. For the anisotropic system we have $\left.S<v_{x}^{2} / v\right\rangle=8 \pi[\operatorname{Im} G(E ; 0,0)-\operatorname{Im} G(E ; 2,0)]$ and $S<v_{y}^{2} / v>=8 \pi t^{2}[\operatorname{Im} G(E ; 0,0)-\operatorname{Im} G(E, 0,2)]$. For $\mathrm{E}=0$ we can show $[13]$ that $\operatorname{ImG}(0 ; 0,0)=\mathrm{K}(\mathrm{t}) / \pi$, $\operatorname{ImG}(0 ; 0,2)=\left(2-\mathrm{t}^{2}\right) \mathrm{K}(\mathrm{t}) / \pi t^{2}-2 \mathrm{E}(\mathrm{t}) / \pi t^{2}$ and $\operatorname{ImG}(0 ; 2,0)$ $=\mathrm{K}(\mathrm{t}) / \pi-2 \mathrm{E}(\mathrm{t}) / \pi$, where $\mathrm{K}$ and $\mathrm{E}$ are the complete elliptic integrals of the first and second kind respectively. In the limit of small coupling $\mathrm{t} \rightarrow 0$, we obtain that $S<v_{x}^{2} / v>\simeq 8 \pi / \hbar$ and $S<v_{y}^{2} / v>\simeq 4 \pi t^{2} / \hbar$. By using these limiting values, we obtain the geometric value $\bar{\sigma}_{0}=\left(\sigma_{0 x} \sigma_{0 y}\right)^{1 / 2}$ of the two conductivities is equal to $\bar{\sigma}_{0}=15 \sqrt{2} t / \pi W^{2}$, where we have used the fact that the relaxation time $\tau=\hbar / 2 \Sigma_{2}=15 \hbar / W^{2}$ in the weak scattering limit.

[15] E. N. Economou, Green's Functions in Quantum Physics, 2nd ed. (Springer, Heidelberg, 1983).

FIG. 1. The numerically determined scaling function for the $2 \mathrm{D}$ anisotropic system for different anisotropic constants $\mathrm{t}$, different energies $\mathrm{E}$ and disorder $\mathrm{W}$. The solid line through the data is the $2 \mathrm{D}$ isotropic scaling function. The $\mathrm{y}$-axis is $\xi_{j} \lambda_{i}\left(M_{j}\right) / \xi_{i} M_{j}$, while the $\mathrm{x}$-axis is $M_{j} / \xi_{j}$. The index i and $\mathrm{j}$ can be either the parallel or the perpendicular direction, respectively.

FIG. 2. The conductance $\mathrm{G}$ in units of $e^{2} / h$ of an anisotropic system $M x N$, versus $M$ for $t=0.1$ and $E=0$. Notice that $\mathrm{G}$ along the two directions is exactly the same.

FIG. 3. The product of the Fermi surface $S_{f}$ with the geometric mean of the localization lengths $\left\langle\xi_{\perp} \xi_{\|}>^{1 / 2}\right.$ is plotted versus the geometric mean of the bare conductivities $<\sigma_{0 \perp} \sigma_{0 \|}>^{1 / 2}$ for all the energies E, t's and W.

FIG. 4. The ratio of $\xi_{\perp} / t \xi_{\|}$is plotted versus $1 / \mathrm{W}$, for $\mathrm{t}=0.1,0.3$ and 0.6 with $\mathrm{E}=0 . \quad \xi_{\perp}$ and $\xi_{\|}$are the localization lengths along the two propagating directions. The solid symbols are the numerical results, while the open symbols are the CPA results. In the insert the numerical results of $\xi_{\perp} / t \xi_{\|}$ versus $1 / \mathrm{W}$ is plotted for $\mathrm{t}=0.3$ with $\mathrm{E}=0.0,1.5$ and 2.0 . 


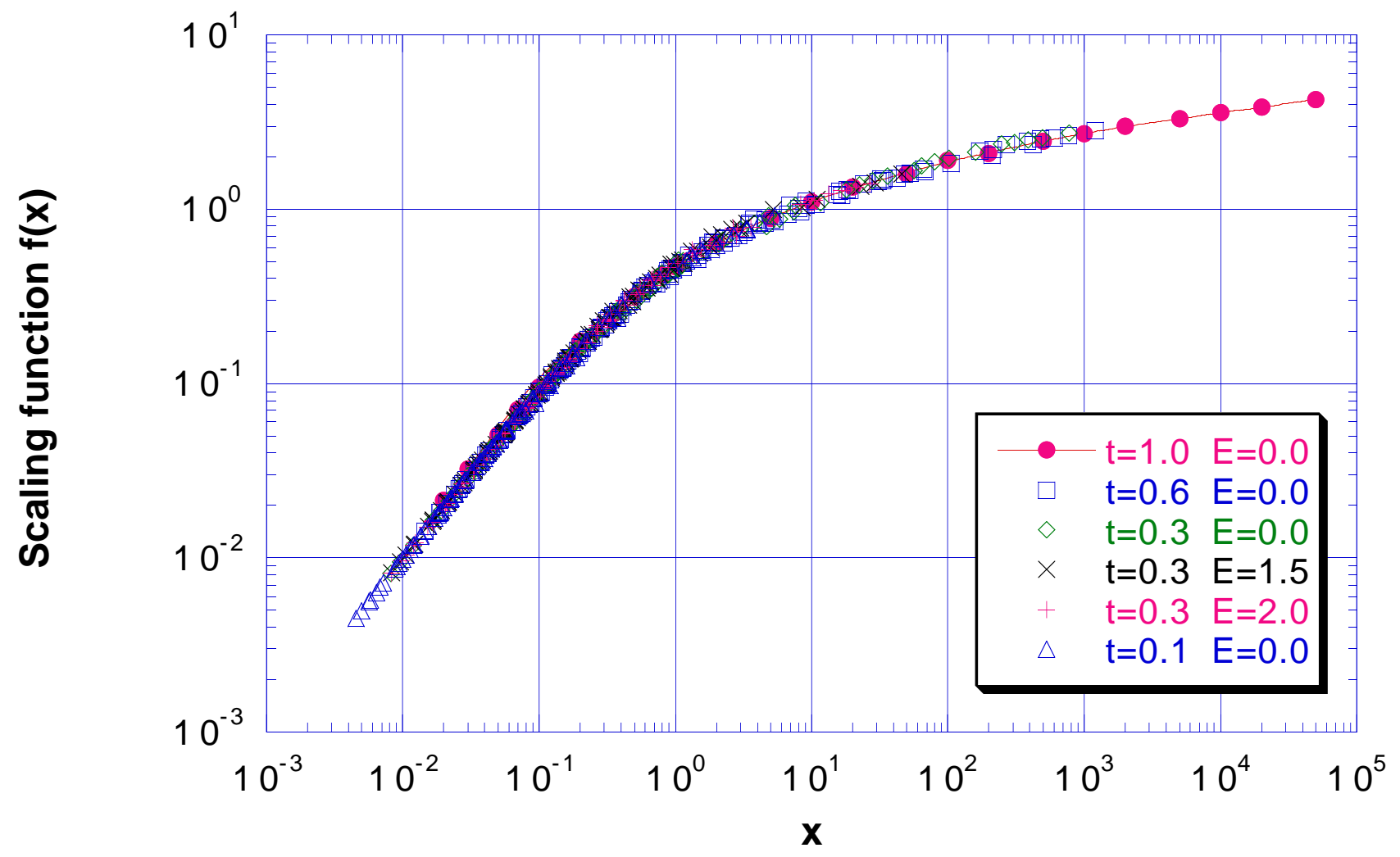




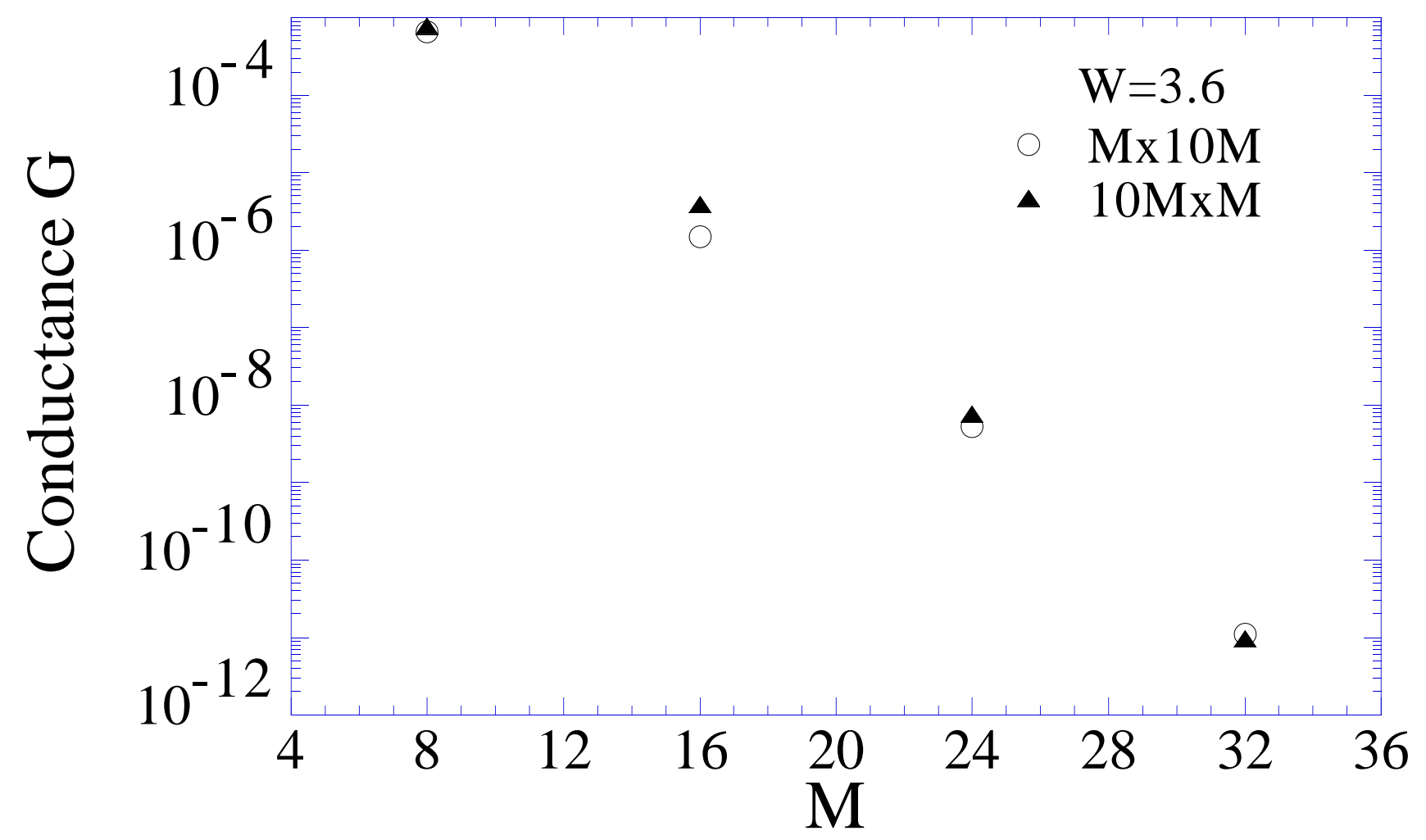




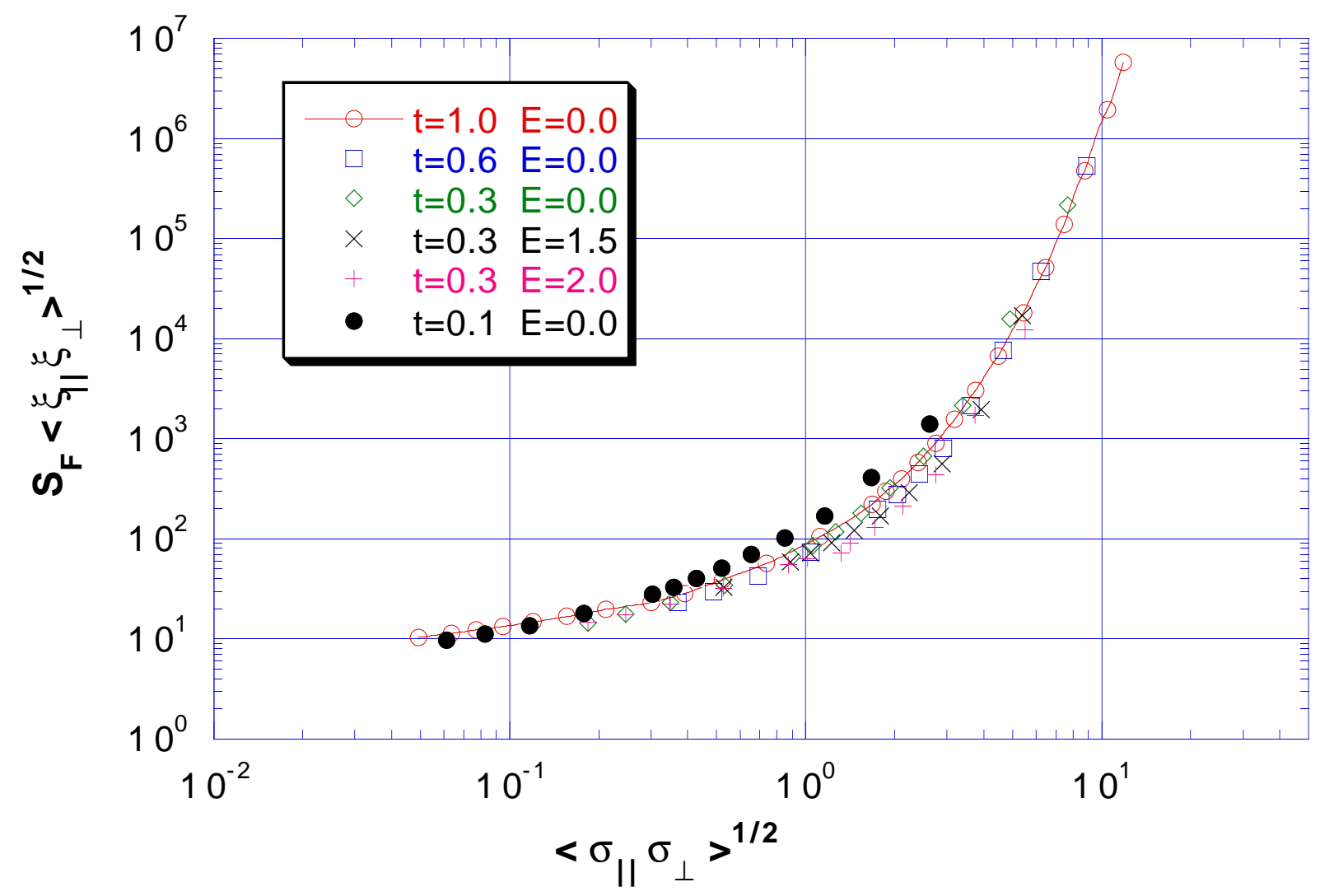




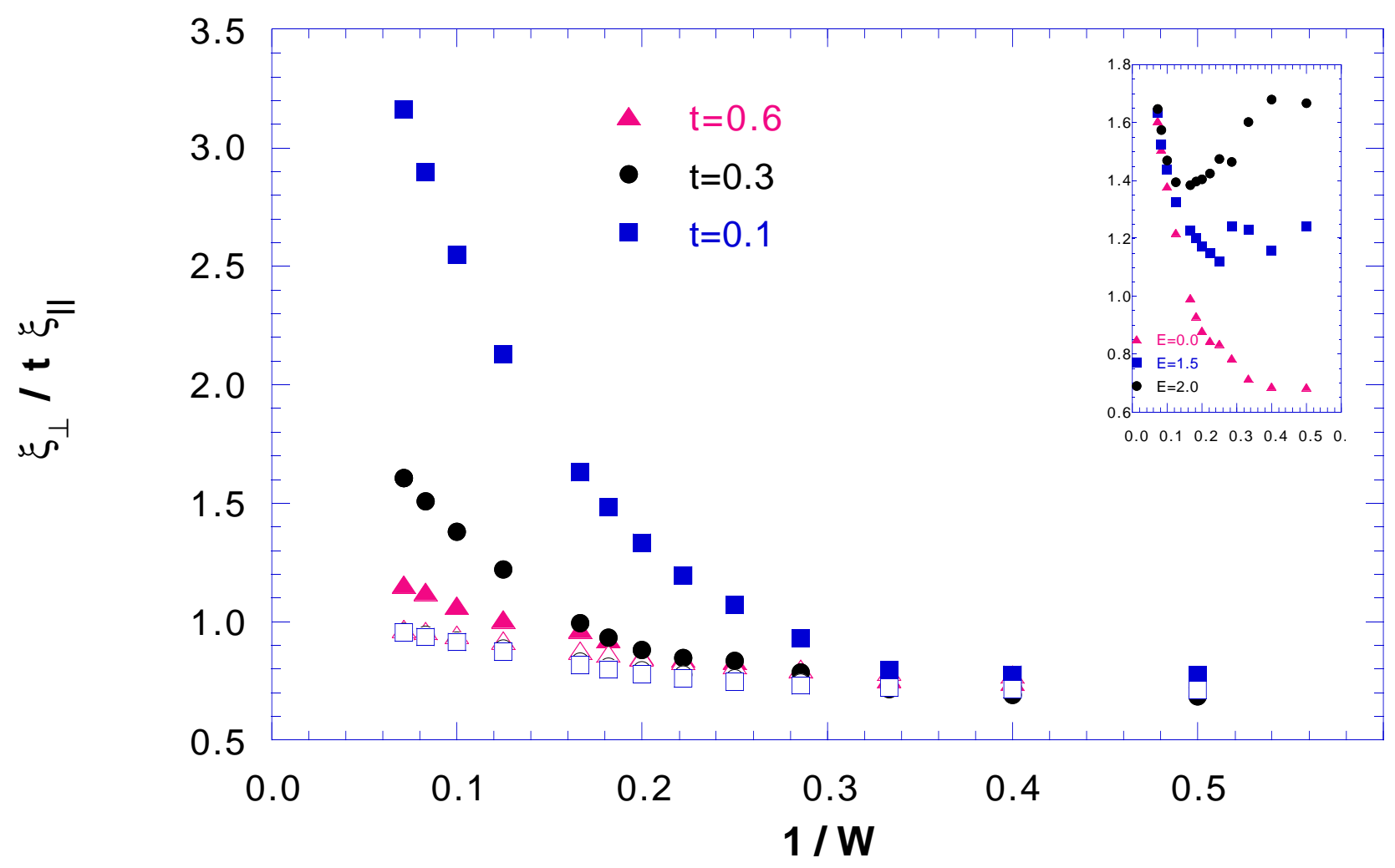


•

ロ

6.

-1
-
-
-
-
0.6 